FORMATION Formation emploi

Revue française de sciences sociales

111 | Juillet-Septembre 2010

Pêle-mêle

\title{
Les « banquiers solidaires » ou la légitimation d'une « profession économique
}

"Socially-conscious bankers" or legitimisation of an "economic profession"

Solidarische Banker oder die Legitimierung eines "Wirtschaftsberufs"

Los "banqueros solidarios" o la legitimación de una "profesión económica"

\section{Pascale Moulévrier}

\section{(2) OpenEdition}

Journals

Édition électronique

URL : http://journals.openedition.org/formationemploi/3103

DOI : 10.4000/formationemploi.3103

ISSN : 2107-0946

Éditeur

La Documentation française

Édition imprimée

Date de publication : 15 septembre 2010

Pagination : $51-65$

ISSN : 0759-6340

\section{Référence électronique}

Pascale Moulévrier, « Les « banquiers solidaires » ou la légitimation d'une « profession économique », Formation emploi [En ligne], 111 | Juillet-Septembre 2010, mis en ligne le 10 octobre 2012, consulté le 30 octobre 2020. URL : http://journals.openedition.org/formationemploi/3103 ; DOI : https://doi.org/ 10.4000/formationemploi.3103 


\title{
Profession
}

\section{Les « banquiers solidaires» ou la légitimation d'une «profession économique »}

\author{
Pascale Moulévrier*
}

\begin{abstract}
Les banquiers de l'économie sociale et solidaire, même s'ils s'autonomisent de la profession de banquier classique, puisent dans le champ économique et financier les ressources pour en devenir une catégorie spécifique. Ils s'apparentent ainsi davantage aux professionnels de la banque qu'aux professionnels du social.
\end{abstract}

Les « banquiers solidaires » constituent un groupe professionnel dont l'analyse en cours permet de comprendre qu'il s'autonomise progressivement de la profession de banquier classique. Cependant, il puise dans le champ économique et financier les ressources pour en devenir une catégorie spécifique.

Ce texte s'inscrit dans le prolongement des travaux réalisés sur les carrières des dirigeants du Crédit Mutuel en France (voir Pascale Moulévrier, 2002). Il vise à saisir les caractéristiques des trajectoires sociales et professionnelles des responsables des

\footnotetext{
1 Recherche menée dans le cadre d'un contrat et financée par : Délégation interministérielle à l'innovation, à l'expérimentation sociale et à l'économie sociale (DIIESES) - Secrétariat général pour les affaires régionales (SGAR) - Préfecture des Pays de la Loire. Laboratoires partenaires principaux : le CARTA(Laboratoire de géographie humaine et sociale) UMR CNRS 6590 - CRESS LESSOR (Centre de recherche en sciences sociales-Laboratoire d'économie et de sciences sociales de Rennes) université de Rennes 2 - IEMN (Institut d'économie et de management) université de Nantes - CENS (Centre nantais de sociologie) EA 3260 université de Nantes. Prolongement de la recherche en cours, financée par le Haut Commissariat aux solidarités actives (HCSA).
}

entreprises - récentes - dont l'activité principale est le micro-crédit. Les investigations ont également permis de recenser les pratiques professionnelles quotidiennes

* Pascale Moulévrier est maître de conférences en sociologie à l'IPSA (Institut de psychologie et sociologie appliquées) - UCO (Université catholique de l'Ouest) Angers, et chercheur au CENS /Centre nantais de sociologie) - université de Nantes.

Elle a notamment publié : Moulévrier P., Lazuech G., Penser l'argent, Ed. Pleins Feux là paraître en novembre 2010). Moulévrier P., Lazuech G., "Les usages sociaux de l'argent : les "démunis" ontils quelque chose à nous apprendre? », in E. Baumann, L. Bazin, P. Ould-Ahmed, P. Phélinas, M. Selim, R. Sobel (sous la direction de) L'argent des anthropologues, la monnaie des économistes, Questions contemporaines, l'Harmattan, 2008. Moulévrier P., Le mutualisme bancaire, Rennes, PUR, 2002. 
d'individus. Leur ancrage professionnel, à cheval entre l'espace de la finance, de la banque et celui du travail social, ne leur confère pas une identité professionnelle et statutaire immédiate. Cela a été observé maintes fois dans plusieurs secteurs d'activités où les dirigeants sont d'abord des responsables, managers, cadres, directeurs (c $f$. notamment Boltanski, 1982), etc., avant d'être banquiers, ingénieurs, etc. Ce point devient ici intéressant à interroger, tant ce flou est constitutif d'une position possible. En effet, les frontières poreuses entre le contenu solidariste des activités et son aspect financier, entre le salariat et le bénévolat, entre le responsable (rarement appelé directeur), les autres employés et les adhérents (bénéficiaires ou pas) fondent cet espace professionnel du micro-crédit. Ainsi, loin de constituer un obstacle pour l'organisation, cette porosité permet aux responsables de mobiliser l'un ou l'autre des aspects de leur activité en fonction des espaces sur lesquels ils interviennent. Ainsi, avec les partenaires sociaux, ils mettent plutôt en avant l'attachement aux dispositifs d'accompagnement ; tandis qu'ils mobilisent davantage la rigueur dans la démarche d'octroi de crédit. La gestion des équipes profite également de cet " entre-deux ». Le " solidaire » confère en effet du sens à un métier (la banque) qui, parfois aux yeux mêmes de ses salariés, en est dépossédé ; la « finance » conserve quant à elle à l'activité une technicité susceptible de leur fournir une identité professionnelle détachée et autonome de celles des travailleurs sociaux.

Cette contribution s'inscrit par ailleurs en rupture avec les analyses sociologiques et socio-économiques des activités d' " économie sociale et solidaire »; ces dernières étant en effet souvent pensées comme susceptibles de fournir une alternative au capitalisme. Ce secteur a été conceptualisé par nombre de chercheurs $^{2}$, dans le prolongement des travaux de Karl Polanyi, comme un «monde à part». Il serait à la fois une forme spécifique d'une "économie plurielle » (Polanyi, ([1944] 1983) et permettrait le «renforcement de la cohésion sociale» (Laville, 1995).

\footnotetext{
2 Pour exemple, en 1996, dans «Vers un nouveau contrat social », Bernard Eme, Jean-Louis Laville, Daniel Mothé, Bernard Perret et Guy Roustang affirment ce positionnement d'entre-deux entre science et militantisme et s'imposent progressivement comme ressources « théoriques » pour l'ensemble d'un secteur «ESS » qu'ils participent largement à constituer comme «monde singulier ». Voir notamment à ce sujet, Fanny Darbus (2009).
}

Délaissant globalement cette approche, nous envisageons le micro-crédit comme profitant d'un nouvel âge, d'un «nouvel esprit» du capitalisme (Boltanski, Chiapello, 1999) ; ce nouvel esprit repose sur la valorisation de la contribution positive de l'entreprise pour la collectivité en termes de lutte contre l'exclusion et de respect des droits fondamentaux. Le prêt contracté dans un organisme solidaire renvoie aux mêmes pratiques qu'un prêt bancaire classique (calcul du ratio de risques, élaboration d'un système de garantie, échelonnage de la dette, évaluation du projet d'utilisation des fonds). Les activités, prises en charge la plupart du temps par des professionnels de la finance, relèvent, dans les deux cas, de la comptabilité, de l'évaluation par bilan comptable.

Ici, ce sont les trajectoires et les caractéristiques sociales des agents qui, historiquement, inscrivent leurs institutions dans des espaces différentiables. En effet, au fur et à mesure de l'élaboration de cadres juridiques adaptés et du partage des populations dont ils ont la charge, ces agents vont octroyer une place singulière à leurs institutions. Ainsi, les grandes banques commerciales (BNP, Société Générale, LCL, Crédit agricole) conservent une clientèle plus urbaine, socialement et économiquement plus dotée ; les banques coopératives (Crédit Mutuel, Banques populaires, Caisses d'épargne, Crédit agricole) s'attachent une clientèle plus modeste tant pour les particuliers que pour les entreprises ; la Poste (devenue Banque postale) demeure la banque des plus pauvres et des plus précaires. Les institutions de finance solidaire deviennent les établissements de crédit des individus non solvables eu égard aux critères utilisés par l'ensemble des banques. En ce sens, les entreprises évoquées ici, qu'elles soient historiquement apparentées aux entreprises capitalistes ou au contraire aux institutions d'《ESS » (économie sociale et solidaire), ont en commun la comptabilité et la morale ; ou, comme le dirait Laurence Fontaine (2008), sont toutes "porteuses de valeurs - fussent-elles libérales », toute économie s'inscrivant dans le politique. Mais alors que les premières pensent le marché comme moyen de produire de la richesse tant économique (production de biens et de plus-value financière) que sociale (création d'emplois et bien-être des populations), les secondes revendiquent des volontés de « faire de l'économie autrement ». Pour autant, l'« économie sociale et solidaire » est consubstantielle au capitalisme. Elle se nourrit en permanence des critiques qui lui sont 


\section{Encadré 1}

\section{Finances solidaires : bref état des lieux}

Les données de cadrage sur le micro-crédit en France sont rares ${ }^{(1)}$. Elles distinguent le micro-crédit professionnel (destiné initialement à financer la création de micro entreprises, d'un montant de 5000 à 125000 euros) et le micro-crédit social (destiné au financement des projets personnels, d'un montant de 300 à 3000 euros - 12000 euros en cas d'accident de la vie); ces deux types de prêts étant prioritairement octroyés aux individus non solvables du point de vue des banques classiques lles "exclus bancaires $\gg|2|$.

Le micro-crédit professionnel s'est développé, en France, à la fin des années 80. L'Adie (Association pour le droit à l'initiative économique), avec ses 130 agences, figure parmi les plus anciennes et les plus actives (un peu plus de 10000 prêts par an). À titre d'exemple, dans les Pays de la Loire, en 2008, trois autres établissements proposaient ce type de prêts : le Crédit Municipal, le Fondes ${ }^{(3)}$ et les Cigales (Clubs d'investisseurs locaux au service du développement d'entreprises solidaires).

Depuis 2005 et le Plan de cohésion sociale ${ }^{(4)}$, le micro-crédit social s'est également déployé, bénéficiant d'une dotation de l'État de 73 millions d'euros sur cinq ans. Portés principalement par des acteurs associatifs et par des institutions sociales locales (Centre communal d'action sociale - CCAS -, Maison de l'emploi, etc.l, ces prêts sont octroyés par des banques partenaires et garantis à $50 \%$ par le Fonds de cohésion sociale.

Cet article repose sur l'enquête par entretiens conduite auprès des "professionnels » du micro-crédit. Au-delà, des analyses des dossiers de prêts, instruits par le Crédit Municipal de Nantes, en collaboration avec les CCAS de Nantes et Angers, et par le "Parcours confiance " (du nom des agences dédiées au micro-crédit appartenant au groupe Caisses d'Épargne) des Pays de la Loire ont montré que les emprunteurs sont majoritairement des personnes seules (près de $80 \%$ ), des demandeurs d'emploi (un peu plus d'un tiers), dont le "reste à vivre » après micro-crédit est de l'ordre de 445 euros. Enfin, le Plan de cohésion sociale stipulait que le micro-crédit ne devait pas servir à financer les fins de mois difficiles, ni à octroyer des fonds destinés à payer les mensualités des crédits revolving. L'étude montre que $31 \%$ des prêts sont utilisés pour fournir de la trésorerie contre $36 \%$ pour le transport lachat de véhicule principalement), 11 \% pour le logement (équipement mobilier notamment) (Source : Traitement par P. Glemain, K. Bachar, in Rapport fimosol, Analyse interdisciplinaire des expérimentations locales du micro-crédit social, juillet 2009.

(1) Le rapport 2008 de l'Agence nouvelle des solidarités actives montre que le nombre de crédits octroyés est faible (2 436 au 31.12.2007) eu égard aux sommes allouées et aux « 3,5 millions d'allocataires de minima sociaux » recensés en 2005 et aux « 8 millions de personnes pauvres », Ansa, Micro-crédit social. Diagnostic et perspectives de développement, mars 2008.

Le travail coordonné par Georges Gloukoviezoff pour le Secours catholique et qui vise à répertorier les formes du micro-crédit social en France offre peu de repères statistiques (cf. Gloukoviezoff et alii, 2008). Le premier rapport (2009) de l'Observatoire de la micro finance, mis en place en 2006, avait pour objectif principal d'évaluer l'application du volet micro-finance du plan de cohésion sociale. Il évoque 51,2 millions d'euros garantis par le Fonds de cohésion sociale pour le micro-crédit professionnel (soit 23000 emplois) et 3,6 millions pour le micro-crédit social.

(2) Voir également Gloukoviezoff (2004) concernant l'émergence de cette catégorie à la fois politique, sociale et conceptuelle d' « exclus bancaires ».

(3) Le Fondes (Fonds de développement solidaire), créé en 1997 dans les Pays de la Loire avec le soutien des collectivités territoriales et des pouvoirs publics, participe au financement de la reprise, à la création et au développement d'activités de « porteurs de projets » dans le cadre de l'entreprenariat classique ou de l'économie sociale et solidaire (associations, coopératives, entreprises adaptées, entreprises d'insertion, etc.).

(4) Ce plan institue, notamment, un fonds, géré par la Caisse des dépôts et consignations. Ce fonds est destiné, aux termes de l'article 80-III de la loi, à : « Garantir à des fins sociales des prêts à des personnes physiques ou morales et des prêts à des chômeurs ou titulaires de minima sociaux créant leur entreprise [...] (et à) prendre en charge des dépenses d'accompagnement des bénéficiaires, liées à la mise en œuvre des projets financés par les prêts qu'il garantit ». 


\section{Encadré 2}

\section{Précisions sur l'enquête et ses méthodes}

L'enquête est réalisée au sein du groupe de recherche FIMOSOL (Finances et monnaies solidaires), dirigé par Pascal Glémain ; le premier volet est financé par la Délégation interministérielle à l'innovation, à l'expérimentation sociale et à l'économie sociale (DIIESES) ; le second par le Haut-Commissariat aux solidarités actives et à la jeunesse (HCSAJ) et la DIIESES : "Le(s) territoires des finances solidaires : une analyse régionale en Bretagne et Pays de la Loire», novembre 2007 et "Analyse interdisciplinaire des expérimentations locales du micro-crédit social », juillet 2009.

Ceux qui sont nommés ici les responsables ou les dirigeants sont le plus souvent des salariés. Pour autant, dans le cas d'associations très récemment implantées, de structures nationales qui testent la viabilité d'un territoire du point de vue du développement de ces activités, ou encore de très petites entités au nombre de dossiers volontairement limité, ce responsable peut être bénévole. Les fonctions des uns et des autres varient surtout suivant le nombre de dossiers traités et de personnel rémunéré.

Une vingtaine d'entretiens approfondis ont été réalisés dans la première phase de l'enquête. Ils fournissent le matériau principal pour notre démonstration (de mars à septembre 2007 et de décembre 2008 à février 2009). Cette enquête a pour ambition d'intégrer l'examen des trajectoires professionnelles dans une analyse plus globale des trajectoires sociales. Une attention particulière est portée aux réseaux associatifs, militants, religieux, politiques, dans lesquels ces individus ont été ou sont impliqués et également à leurs formes spécifiques de socialisation familiale et scolaire. Interrogés sur leur quotidien professionnel, ces " banquiers solidaires " nous livrent à la fois leurs rapports au travail et leurs ambitions, tant professionnelles que morales.

Le terrain se limite à la Loire-Atlantique ${ }^{*}$ ) où se côtoient une diversité de structures, eu égard notamment au nombre de dossiers traités et au lien plus ou moins exclusif qu'elles entretiennent avec l'activité de micro-crédit.

La population d'enquête appartient à une multiplicité de structures : principalement, toutes petites associations locales n'appartenant à aucun réseau, pour qui l'activité de micro-crédit est intégrée à une pluralité de missions de réinsertion économique et sociale (l'association Comptoir de vie par exemple) ; associations locales appartenant à un réseau dont elles se réclament à la fois vis-à-vis du fonctionnement et des principes (Les Cigales); associations dont le territoire d'intervention est le département, la région, l'inter-région, autonomes dans la gestion de leurs activités mais rattachées à un groupe national (L'Adie); antennes ou délégations d'associations nationales (La Nef - Coopérative de finances solidaires); services spécifiques de groupes bancaires coopératifs (Le parcours Confiance des Caisses d'Épargne); initiatives régionales ou locales de réseaux bancaires (dont le Crédit Municipal, le Crédit Mutuel, le Crédit Coopératif).

(*) : La Loire-Atlantique constitue le territoire exploratoire de la recherche. Ce choix se justifie, en dehors de la proximité géographique de l'équipe de recherche, par l'ancrage fort des activités de micro-crédit dans le département. Cet ancrage peut être mis en relation avec le développement ancien, et encouragé politiquement ces dernières années, du secteur de l'« économie sociale et solidaire ». Au 31.12.2008, 75 \% des micro-crédits personnels garantis ont été octroyés dans l'Ouest de la France (Ouest, Nord Ouest et Sud Ouest) - Source FIMOSOL (programme de recherche Finances et Monnaies Solidaires), Rapport final HCSAJ-DIIESES, juillet 2009, d'après les données CDC-DDTR (Caisse des dépôts et consignations-Direction du développement territorial et du réseau), Département économie sociale. 
adressées. Il n'y a pas en ce sens d'économie plurielle, moins encore d'économie alternative, mais des «mentalités économiques » différentes (Weber, 1964).

Les décisions politiques et les dispositifs juridiques qui y sont associés pourraient laisser croire à un positionnement marqué de ces " nouveaux métiers » du côté de l'action sociale, de la lutte contre la pauvreté. À l'inverse, nous tenterons de montrer que les «banquiers solidaires » et leurs institutions ancrent plutôt leurs activités dans le champ de la finance. Ils prennent ainsi position sur le marché du crédit, dont le micro-crédit n'est finalement qu'une branche.

Dans un premier temps, nous reviendrons, par la description des tâches, sur un quotidien professionnel largement consacré au crédit. Cependant, notre objectif est de saisir les liens entre les activités présentes et les ressources, sédimentées dans le temps, dont disposent les individus. Cette analyse par les trajectoires permettra d'identifier deux types de « banquiers solidaires » : les premiers, marqués par l'ethos du service public (partie 2); les seconds, à la fois contraints par la nécessité de l'insertion professionnelle et séduits par ce qu'ils considèrent comme un segment « moralisé » du marché financier (partie 3). Pour autant, et malgré la relative hétérogénéité de leurs parcours, tous sont portés, tant en raison de leur frottement quotidien à l'univers financier, de leurs formations, que de leur volonté de reconnaissance et de carrière, à croire en l'économie et finalement à participer à l'étiquetage économique de leur «profession » (partie 4).

Les responsables rencontrés constituent donc un groupe relativement hétérogène en termes de statuts (salariés et, dans une moindre mesure, bénévoles), de fonctions (directeur - dans le cas des associations autonomes, président - dans le cas des associations sans salariés, chargé de mission - dans le cas des structures rattachées à des groupes nationaux), d'activités (management, gestion, finance, accompagnement, montage de dossiers). Pour autant, l'analyse de leurs trajectoires et de leurs discours permet de les envisager comme communauté ${ }^{3}$; communauté qui

\footnotetext{
3 Même si la " cohésion » de l'espace de la micro-finance ne peut être attribuée à ce que Max Weber appelle notamment « la communauté de compréhension ", l'usage d'un langage commun, d'une terminologie de la « finance solidaire » participe à rassembler les individus derrière l'idée du « travailler autrement », du « travailler ensemble », « pour les autres ».
}

est cimentée par des dispositions à la mise en œuvre d'une « économie moralisée » (Moulévrier, 2002, chap. 6) et qui partage un même registre d'activités : le crédit aux «plus pauvres ».

En raison de leur place singulière dans le champ bancaire et des publics concernés, le service proposé par ces entreprises comporte une double dimension, financière et d'accompagnement. Les dirigeants composent alors, tant dans leurs parcours que dans leurs discours, un rôle hors du seul registre bancaire, une figure en rupture avec l'économie marchande. Toutefois, par leurs activités et leurs goûts pour la " chose financière ", ils dessinent davantage les contours d'une « profession économique » que ceux d'une « profession sociale ».

Enfin, la forme le plus souvent associative de ces institutions renforce le sentiment de participer à une autre économie, de promouvoir, en s'engageant au-delà de la seule logique professionnelle, un autre modèle de société, voire de construire pour soi. Ainsi, ce directeur d'une association de finances solidaires revendique une identité professionnelle singulière : «Moi ce que je suis, ça va vous paraître un peu prétentieux mais c'est difficile de qualifier notre boulot... Moi je suis cadre dirigeant, particulièrement du secteur associatif. Je connais les partenaires, les rouages, les leviers, les modes d'intervention. Je peux vendre aujourd'hui de l'économie sociale et puis demain autre chose... du handicap. Je suis un généraliste $d u$ management des entreprises associatives ».

\section{LE « CRÉDIT SOLIDAIRE » : DES PRATIQUES BANCAIRES $E N$ « SOURDINE »}

Pour rappel, les institutions françaises de micro-crédit évoquent régulièrement leurs inspirations internationales, et notamment leur lien conceptuel plus ou moins ténu avec la banque de Muhammad Yunus au Bangladesh. Cette initiative est officiellement mise en place en 1977, au Bangladesh, sous le nom de « Grameen ». Dès le milieu des années 80 , dans les pays du sud d'abord puis en Occident, elle va effectivement 
contribuer, au moins symboliquement, au développement de l'espace des «finances solidaires $»^{4}$.

Au-delà de cette dimension internationale, les établissements français proposant du micro-crédit (pour la création de petites entreprises ou pour les projets d'une clientèle de " particuliers », pour reprendre le langage bancaire), sont des institutions ancrées dans les territoires locaux (Glémain et alii, 2007). Le statut d'association ou de coopérative renvoie à des modes spécifiques de constitution des entreprises. Souvent en effet, une association ou une coopérative de microcrédit démarre localement grâce à l'investissement bénévole de quelques individus portés politiquement, ou comme ils le disent " humainement », ou encore « comme citoyens », à s'intéresser à la question de l'exclusion bancaire ou plus largement sociale. Ces origines territorialisées et marquées par l'engagement plus que par la compétence professionnelle expliquent en partie le profil des premiers salariés et dirigeants.

Parallèlement pourtant, le rattachement de ces initiatives locales à des réseaux nationaux qui leur préexistent ou qui se sont organisés conjointement et le développement des activités peuvent précipiter le passage à une dimension plus entrepreneuriale. Ils peuvent ainsi conduire au recrutement d'un personnel permanent salarié. ${ }^{5}$

Pour autant, ce processus de professionnalisation n'homogénéise que très progressivement les pratiques. En fonction du type de structure (appartenant à un groupe, comme c'est le cas des différentes agences « Parcours Confiance » des Caisses d'épargne ou bien au contraire association isolée) et du nombre de ses salariés, du volume des demandes de prêts et du volume de crédits, du nombre et du type de partenariats, d'accords et de conventions passés avec l'État, les collectivités territoriales, les banques notamment, les responsables peuvent être amenés à essentielle-

\footnotetext{
${ }^{4}$ Certaines institutions de micro-crédit se réclament de « Grameen bank » de Yunus comme caution d'appartenance certifiée à l'espace des « finances solidaires ». On citera « Planet Finances », présidée en France par Jacques Attali et co-présidée par Mohammad Yunus. L'Adie, lors d'une de ses assemblées générales en mars 2007 , recevra également son soutien officiel.

${ }_{5}^{5}$ Pour certains réseaux plus anciens comme l'Adie, la structure nationale peut décider l'implantation d'une antenne régionale sans qu'il y ait eu nécessairement d'acteurs locaux impliqués en amont. Dans ce cas, les modalités de recrutement peuvent évoluer, tout en gardant des singularités par rapport aux banques.
}

ment diriger l'entreprise et manager les équipes, ou bien également à monter ou superviser les dossiers de demande de prêts et à rencontrer et accompagner les emprunteurs. Pourtant, quel que soit leur mode d'intervention dans l'organisation, ils développent de fait un rapport au travail qui occulte en partie le temps conséquent passé à la gestion de l'entreprise et au montage financier des dossiers de prêts. En effet, non seulement les activités comptables et bancaires sont peu décrites dans les entretiens, mais elles sont, au sein du collectif de travail, peu visibles. Elles sont prises en charge individuellement dans l'isolement du bureau et sont rarement évoquées lors des réunions d'équipe ; celles-ci étant largement consacrées aux modalités d'accompagnement, à la répartition des tâches entre la structure de micro-crédit et les partenaires sociaux et bancaires, aux perspectives de développement de l'activité, et enfin au positionnement « éthique » de l'entreprise. Pourtant, après observations et entretiens, les heures consacrées aux bénéficiaires potentiels représentent $20 \%$, par opposition aux $80 \%$ d'heures consacrées au travail administratif et financier ${ }^{6}$. Plus l'activité est développée (au sens du volume des activités et du nombre de salariés), plus la rationalisation et la bureaucratisation des pratiques s'intensifient, plus les compétences en techniques d'évaluation financière ${ }^{7}$ deviennent, comme c'est le cas pour les commerciaux des agences bancaires, indispensables au fonctionnement des structures.

$\mathrm{Au}$ « Parcours confiance » des Pays de la Loire des Caisses d'Épargne, par exemple, qui, au $1^{\text {er }}$ janvier 2009 , totalisait 318 prêts en cours, le directeur et ses deux collaboratrices traitent les demandes sur la base des dossiers instruits par leurs partenaires (Union départementale des affaires familiales - UDAF, Familles rurales, Secours catholique, Union régionale interfédérale des organismes privés sanitaires et sociaux - URIOPSS, etc.) sans pour autant être en contact avec eux.

\footnotetext{
${ }^{6}$ Par travail administratif et financier, on entend notamment le travail de recherche de subvention de fonctionnement et de partenariat bancaire, la gestion de l'entreprise (humaine et administrative), le montage financier des dossiers de prêts.

7 L'évaluation financière recouvre le calcul de ratio de risque, et éventuellement la mesure du " reste à vivre », eu égard notamment à la potentialité de remboursement, au taux d'endettement, au niveau et à la régularité des revenus.
} 
L'un d'eux décrit son travail : «D'une part, il faut tout lire et la partie la plus importante, c'est l'explication qui est donnée par le partenaire, parce que nous, nous n'avons pas la personne en face de nous, mais la façon de présenter la situation va nous permettre de savoir où en est la personne dans son parcours. Est-ce que c'est un accident de vie? Est-ce qu'elle est en situation d'endettement, parce qu'elle a rencontré des difficultés, elle a perdu son travail ? Moi, je regarde le montant, la durée, l'objet. (...) Notre travail est surtout un travail dossier papier et informatique. Ensuite, en Caisse d'Épargne, il y a la notion de client. Donc, c'est une notion un peu plus large qu'un compte. Donc, on crée ce client, on ouvre des comptes. On crée le prêt. On envoie les offres de prêts, donc, on s'engage à le faire rapidement. "

De leur côté, les dirigeants encore bénévoles des Nef et des Cigales, deux institutions qui traitent peu de demandes par an ${ }^{8}$, consacrent effectivement moins de temps à l'administration des dossiers qu'à l'accompagnement des demandeurs. Mais lorsque les demandes s'accélèrent, l'accompagnement passe souvent à des formes plus efficaces, moins coûteuses en temps et en investissement humain. Le téléphone remplace alors progressivement les rencontres individualisées sur le terrain.

Une correspondante de la NEF, bénévole et intervenant dans l'Ouest de la France, va, dans les six mois à venir, laisser la responsabilité de la délégation à un salarié. L'activité s'est intensifiée et le développement justifie aujourd'hui un bureau ayant pignon sur rue et le recrutement d'un responsable. Elle nous raconte son quotidien de « travail » :

\section{«Comment faisiez-vous et comment faites-vous ? Vous recevez les gens chez vous? »}

« De toute façon, on voit toujours les gens. S'il y a un lieu du projet, si c'est une exploitation agricole ou une ouverture de magasin, on rencontre forcément les gens sur le lieu, de façon à voir le contexte».

\footnotetext{
${ }^{8}$ Pour exemple, une des Cigales observées en Pays de la Loire a monté en cinq ans deux dossiers de prêts pour création d'entreprises. Leur activité principale reste la gestion de l'indivision que les « cigaliers » forment ensemble afin de constituer « une épargne solidaire ». L'antenne régionale de la Nef traite, de son côté, quelques dizaines de dossiers par an, mais s'apparente plus directement à une banque dans la mesure où elle offre à ses adhérentsclients la possibilité d'une ouverture de compte courant. L'épargne réalisée par les sociétaires sert, pour partie, à l'octroi de prêts.
}

\section{«Ca vous prend combien de temps?»}

"Si je fais la moyenne, c'est un mi-temps. Là tout l'hiver, j'étais au bureau tous les matins. Là, chez moi.»

\section{«Sur une année ?»}

"C'est trois heures par jour (...) au jour d'aujourd'hui, c'est 250 contacts au total depuis le début, en sachant que les premières années, il y avait quasiment rien, ça augmente. »

Une responsable de l'Adie (Loire-Atlantique-Vendée), environ 35 ans, titulaire d'une maîtrise en sciences économiques, évoque l'évolution des procédés d'accueil du client :

\section{« Vous avez évoqué le fait qu'à l'époque vous aviez des rencontres physiques? »}

"Ça veut dire qu'on recevait toutes les personnes qui nous appelaient en physique, sans savoir avant si elles pouvaient avoir un prêt Adie, sans savoir où elles en étaient dans le montage de leur dossier, sans savoir même si elles avaient un projet de création, ce qui n'arrive plus maintenant puisque la première étape à l'Adie, c'est un accueil diagnostic téléphonique par des conseillers qui sont formés et qui sont des anciens conseillers en micro-crédit d'ailleurs, qui vont prendre la personne au téléphone pendant un quart d'heure, vingt minutes, le temps qu'il faudra d'ailleurs, parler d'elle, de son projet, savoir où elle en est, prendre quelques renseignements... (...) On est très en amont ... Si la personne a avancé un peu dans son projet et qu'elle est éligible à l'Adie, on peut directement lui prendre un rendez-vous avec le conseiller qui s'occupera de son dossier. »

Les activités des dirigeants des institutions de « finances solidaires » sont donc largement centrées autour du crédit financier d'une part, et de la gestion rentable de leur entreprise d'autre part (par la collecte d'épargne, la recherche de financements publics et privés, la signature de partenariats avec les banques). Pour autant, ces dirigeants revendiquent, chacun à leur niveau, une réalité professionnelle dans laquelle s'équilibrent contenu financier du "métier » et considération humaniste pour la clientèle. Le bénéfice symbolique attaché aux fonctions passe à la fois par la reconnaissance d'une compétence commerciale ou 
gestionnaire et par la croyance partagée au « travailler autrement ».

Ainsi tous s'attachent à décrire précisément, avec les mots justes, ce qui fait la spécificité de leur travail, toujours par opposition implicite au secteur plus ou moins « diabolisé » du tout marchand.

Un directeur du Fondes (Nantes), 44 ans, ex-travailleur social, diplômé en formation continue d'un DESS (diplôme d'études supérieures spécialisées) en sciences économiques (orientation économie sociale et solidaire) décrit son rôle. Son propos est révélateur de la hiérarchie qu'il établit entre les différents aspects de sa fonction, qui privilégie le contenu « éthique » :

«Vous dites que vous pourriez faire ça ou autre chose ?»

«Oui, il y a une limite éthique. Clairement. Je ne veux pas être sur le marché du capital. (...) Je ne suis pas complètement dans "Vivre et travailler au pays" mais j'y suis quand même un peu et en même temps je suis dans quelque chose d'utile. Non, bien sûr je ne postule pas par hasard, ce n'est pas parce que le Fondes cherche quelqu'un que je me dis je vais répondre. (...) Je sors de douze mois de DESS où on ne m'a parlé que de ça. (...) Même si avec le recul, je me dis que j'ai fait ça pendant 20 ans sans le savoir »

Les premières questions relatives à la description des activités suscitent systématiquement une réponse liée à l' « éthique », certains affirmant que ce qui les anime, c'est la volonté «de mettre les gens ensemble », de « se mettre au service de l'engagement », de défendre « cette solidarité, cette proximité pour la décision, pour le choix, pour la confiance et l'échange », ou de " dire que l'humain est au cour de [leur] être, que c'est l'autre qui [les] construit $»{ }^{9}$

Cette revendication humaniste ne doit pourtant pas masquer la réalité professionnelle quotidienne et les activités financières qu'elle comporte. Très vite d'ailleurs, les compétences économiques vont s'imposer comme nécessité pour le développement des structures ; compétences que les institutions acquerront soit par la formation des personnels déjà en place, soit par le recrutement de diplômés en gestion, banque

9 Extraits d'entretiens réalisés de mars à septembre 2007 et de décembre 2008 à février 2009. et finances ; soit, pour les banques notamment, par l'affectation sur ces postes « solidaires » de salariés expérimentés de leur propre réseau bancaire.

Mais au-delà de cette inclinaison générale à inventer au quotidien des manières " humaines » d'être banquier, il est possible de repérer à la fois deux générations et deux profils principaux de responsables de structures de micro-crédit.

\section{RETOURNER À L'ÉCOLE, QUITTER LA FONCTION PUBLIQUE}

La trajectoire professionnelle des responsables salariés ou bénévoles interrogés nous éclaire en effet sur leurs dispositions à adopter une posture convaincue de « banquier éthique ».

En dehors d'un constat portant sur l'expérience commune à tous d'une forme de "rupture $»^{10}$, un premier groupe, majoritaire, possède des expériences dans des domaines éloignés de la finance. Enseignants, instituteurs, travailleurs sociaux, aides à domicile, cadres de la fonction publique sont autant de professions exercées par les dirigeants dans une première partie de leur carrière. Dans ces étapes antérieures, ils ont accumulé des dispositions éducatives facilement transposables à " l'encadrement » économique des catégories populaires.

Pour ceux-là, l'arrivée sur le marché du travail des « finances solidaires » succède à une pause consacrée le plus souvent à un temps de formation universitaire. Comme ils l'évoquent eux-mêmes, le premier métier a épuisé un certain nombre de leurs motivations initiales. Conjugué à leurs divers engagements associatifs, il a suscité des « envies de connaissances » et de mobilité ascendante et des « besoins » d'exercer des fonctions à responsabilité. Il en va ainsi des dirigeants des entreprises d'insertion par l'économie qu'évoque

\footnotetext{
${ }^{10}$ Cette rupture dans les histoires professionnelles des personnes rencontrées fait écho aux accidents de la vie dont sont souvent " victimes » les bénéficiaires, même si elle n'est pas comparable aux ruptures qui traversent les dossiers de demande de prêts des bénéficiaires (perte d'emploi, faible qualification, divorce et séparation conflictuelle, maladie, handicap). Les individus enquêtés ont en commun avec les bénéficiaires du crédit l'expérience du changement.
} 
Gilles Lazuech (2006), « les trajectoires professionnelles de ces personnes sont souvent non linéaires, et pour certaines, s'apparentent à une véritable ascension sociale»; ascension sociale concrétisée ici par le passage d'une profession intermédiaire de la fonction publique à des postes de cadres du secteur associatif, à des fonctions de « manager-militant».

Un directeur d'une association de « développement solidaire », qui octroie des micro-crédits professionnels, déclare :

"Je ne sais pas si c'est atypique dans l'économie sociale et solidaire parce que je le retrouve chez d'autres de mes partenaires mais... moi je suis un travailleur social à l'origine. J'ai fait une formation de travailleur social. J'étais animateur socioculturel mais intervenant dans des quartiers "politique de la ville", avant de devenir directeur de centres sociaux et d'action sociale en passant par des foyers de jeunes travailleurs. Après 20 ans, j'en ai eu un peu marre pour être honnête. En tout cas, je sentais qu'il fallait que je tourne la page sans vouloir la tourner complètement. Il fallait de la conviction dans mon travail. Comme beaucoup à cet âge, vers 40 ans, j'ai rebondi par une formation qualifiante et j'ai fait un DESS (diplôme d'études supérieures spécialisées) en économie du développement local à l'UFR (Unité de formation et de recherche) de gestion dont je suis sorti immédiatement pour entrer au Fondes »

Ce passage par la formation continue ne correspond pas uniquement au modèle classique du congé individuel de formation dont certains profitent. Il peut également s'apparenter à des processus plus longs. Ainsi, l'accroissement des responsabilités dans le secteur associatif permet au salarié de négocier avec l'employeur pour obtenir des actions de formation ponctuelles avec un passage à temps partiel ou un détachement.

Un gérant de Cigales, bénévole, bénéficiant d'un temps partiel choisi et négocié dans sa fonction de cadre territorial, illustre bien ce type de trajectoire :

"Je suis ingénieur travaux publics. J'ai suivi des formations en géographie et puis j'étais dans une structure d'études qui travaille sur Bretagne et Pays de Loire, donc c'est un parcours ; j'ai fait dix ans là dedans. Aujourd'hui (...) je suis à la DDE (direction départementale de l'Équipement). Je suis dans l'opérationnel. Par ailleurs, $j$ 'ai un parcours associatif. (...) C'est tout un travail et c'est vrai que dans cette construction collective, il y a beaucoup de choses qu'on apprend, il y a beaucoup de manières d'être qu'on apprend aussi. En 98, je suis rentré à la DDE. (...) Dans Les Cigales, moi, je suis récent. Je suis rentré il y a quatre ans en 2003. Depuis septembre, je suis à $80 \%$ à la DDE. J'avais déjà été à $80 \%$ pour me permettre de faire la formation à la fac et après pour mes engagements associatifs et en 98 , en rentrant à la $D D E$, j'ai repris à $100 \%$, c'était dans le contrat. Là il y a eu une réorganisation de la DDE; en plus, je savais que je trouvais à l'extérieur plus de valorisation que dans le milieu professionnel.»

Il est intéressant de noter que les carrières de la plupart des responsables ${ }^{11}$ des institutions de micro-crédit se sont déroulées dans la fonction publique (Éducation nationale, fonction publique territoriale, Action sociale). La souplesse (détachement, congé, mise en disponibilité, retraite anticipée) du statut de fonctionnaire a favorisé ces reconversions qui, dès lors, sont sans risque du point de vue de la perte d'emploi.

Pour autant, cet élément n'est jamais évoqué comme tel. Ce que les individus retiennent de leurs expériences professionnelles antérieures de fonctionnaire, d'agent de l'État, d'acteurs de la mise en œuvre de l'intérêt général renvoie plutôt à l'immobilisme des institutions publiques. Le manque de moyens (humains et financiers surtout), la place grandissante accordée aux tâches administratives sont autant d'arguments qui ont fait obstacle à leur « volonté d'agir sur les choses ».

Ce bilan négatif de leur fonction antérieure contribue à construire l'espace des entreprises solidaires comme une alternative possible à l'État et au marché. Possible car débarrassée - dans leurs esprits - des lourdeurs de la bureaucratie publique ; mais possible aussi car pensée comme éloignée des logiques de l'intérêt privé, du gain et de la rentabilité. Les entreprises de microcrédit deviennent des lieux d'exercice de l'initiative solidaire; des lieux où l'économie et l'intérêt général sont conjointement mis en œuvre par des individus

11 Toutes les personnes rencontrées en entretien et en situation de reconversion professionnelle étaient issues de la fonction publique. 
localement investis et dès lors convaincus de leur « utilité sociale » (Hély, 2008).

La dimension réduite des actions, le déploiement des activités sur des territoires locaux et les petites équipes professionnelles ${ }^{12} \mathrm{~s}$ 'avèrent également fondamentaux dans l'adhésion des responsables à l'entreprise associative.

\section{UN ESPACE PLUS OU MOINS CONVOITÉ D'INSERTION PROFESSIONNELLE}

Les plus jeunes recrutés, c'est-à-dire les moins de 40 ans, sont tous diplômés en sciences économiques, gestion, banque, finances ou commerce. Pour autant, une majorité d'entre eux ont souvent une trajectoire moins linéaire que leurs homologues (générationnels et professionnels) recrutés dans le secteur bancaire classique.

Titulaires au mieux d'un bac +3 à bac +4 , ils ont très souvent mis un terme rapide à leur projet initial de formation. Poussés par des contraintes matérielles, ils ont dû accéder rapidement à l'emploi (leurs parents appartiennent plutôt aux catégories populaires supérieures) ; dès lors, ils s'orientent vers un secteur professionnel qui leur apparaît en adéquation avec des compétences, eu égard à l'interruption des études (en licence, BTS - brevet de technicien supérieur-, ou maîtrise), partiellement acquises. Pour eux, travailler dans la finance solidaire mobilise moins de savoirs technico-financiers exigés dans les banques ou les entreprises financières « classiques »; savoirs qu'ils auraient pu acquérir dans des masters professionnels. Le monde de l'économie sociale et solidaire apparaît alors comme un espace professionnel moins économique, moins financier ; un espace où d'autres qualités - apparentées à des compétences sociales pourront être mobilisées.

\footnotetext{
12 Ces caractéristiques pouvant également être comprises comme autant de différences avec le service public, même dans ses dimensions les plus décentralisées.
}

Une responsable de l'Adie (Loire-AtlantiqueVendée), 35 ans, titulaire d'une maîtrise en sciences économiques, déclare :

«Moi je suis à l'Adie depuis pratiquement cinq ans. J'ai débuté comme conseillère en micro-crédit sur la ville de Rennes et je suis devenue responsable Loire Atlantique Vendée, depuis un an et demi maintenant. J'ai intégré l'Adie, c'était pratiquement mon premier travail après mes études. Ma candidature était une candidature spontanée que j'ai envoyée à l'Adie parce que j'avais des diplômes en sciences économiques et en gestion et que je voulais travailler dans une structure qui touchait à l'économie sociale et solidaire tout simplement. (...) Mon diplôme le plus haut c'est une maîtrise en sciences économiques passée à Nantes. Avant, j'étais à Vannes. (...) J'avais, d'ailleurs, c'est assez contradictoire, été présélectionné pour un DESS en communication économique sur Aix-en-Provence mais je n'ai pas pu le faire : les frais d'inscription étaient très élevés et le matériel qu'on nous demandait d'avoir en cours était très cher aussi. Donc en dehors de ce que je pouvais faire, puisque j'avais déjà payé mes études par des prêts (...) et donc j'ai décidé de commencer à travailler et ensuite de reprendre à bac +5 quand j'en aurai envie. »

« D'où vous est venue cette envie de postuler à l'ADIE ? »

«Moi quand j'étais enfant ils [les membres de sa famille] étaient dans un milieu ouvrier et parfois il $y$ avait des problèmes d'argent également, je pense que ça joue. Après, quand nous étions étudiants, parce qu'on est quand même trois enfants, en tout cas mon grand frère et moi, quand on a continué nos études, l'entreprise de mes parents commençait juste. Je pense que j'étais parfois en décalage avec les personnes qui étaient avec moi en cours. Ils étaient d'un milieu très aisé alors que moi j'étais d'une classe moyenne. Et puis je pense que de voir que ma mère qui n'avait pas de possibilité de voie de réinsertion classique pouvait quand même s'en sortir très bien grâce à son entreprise... En allant à l'Adie, je savais de quoi on parlait. »

Parmi ces plus jeunes professionnels de la finance solidaire, on retrouve également de plus en plus souvent des diplômés des grandes Écoles - de commerce notamment. Ils ont choisi, en fin de cursus, une option, 
un parcours, voire un master orienté « économie sociale et solidaire $»^{13}$. Certes, par leur choix de spécialité, ces étudiants construisent, au sein de l'école, une figure singulière en rupture avec la masse des étudiants formés plus directement à l'économie du capital. Cependant, ils n'en demeurent pas moins sensibles à la nécessité de « faire carrière » et à la question des revenus. Au sein des entreprises associatives ou coopératives de finances solidaires, les possibilités d'accéder rapidement à des postes à responsabilité et à des revenus élevés (tels que le laisse entrevoir le cursus en grandes Écoles) restent limitées. Dès lors, elles favorisent le départ rapide de ces " nouvelles recrues », soit vers les institutions internationales (ONG - organisations non gouvernementales - notamment) soit in fine vers les entreprises classiques, notamment dans les services « développement durable » ou plus récemment dans des fonctions en lien avec la responsabilité sociale des entreprises (RSE).

\section{L'INTÉRÊT POUR L'ÉCONOMIE}

Ainsi, les dirigeants des entreprises de finances solidaires ont des carrières moins linéaires que celles des cadres supérieurs des institutions bancaires et financières traditionnelles. Pour autant, ils ne marquent pas les fonctions de direction d'une empreinte résolument socio-éducative et d'ambitions exclusivement humanistes.

Leurs motivations individuelles à s'orienter vers les formations supérieures en économie (offrant des enseignements de fiscalité, comptabilité, théories économiques, gestion, management, stratégies, etc., potentiellement appliqués à l'économie sociale et solidaire), à chercher un poste ou une activité dans le secteur du micro-crédit, à « faire carrière » dans

\footnotetext{
13 Citons la création, en 2006 à HEC (Hautes études commerciales) Paris d'une Majeure "Alternative Management » qui « s'adresse à des étudiants qui souhaitent contribuer au renouvellement des pratiques managériales en favorisant une plus grande prise en compte des aspects sociaux, éthiques et environnementaux » (cf. http://alternative.hec.fr); ou encore la spécialisation en Arts, NGO's and Philanthropy Management de l'EDHEC (École des hautes études de commerce) qui permet aux diplômés de " $s$ 'insérer entre autres dans les organisations non profitables ou le secteur public » (cf. http://programme.edhec.com/master.php).
}

la finance quand elle est « solidaire », traduisent un intérêt partagé pour la question économique.

Comme dans le secteur bancaire coopératif et mutualiste, les pratiques professionnelles et les discours qui les portent sont marqués, d'une part, par des formes plus ou moins conscientes de dénégation du profit (Moulévrier, op. cit., pp. 103-137) et d'autre part, par un attachement collectif à la mise en œuvre possible d'une économie renouvelée. L'économie, telle qu'elle est envisagée par les responsables des entreprises de micro-crédit social ou plus largement des « finances solidaires », n’est déniée que dans son contenu marchand, dans son acception purement capitaliste, c'est-à-dire dans la considération première d'une auto-expansion délibérée, entretenue, optimale du capital.

Chacun exprime son intérêt pour l'économie et cherche à acquérir des connaissances légitimes en économie - c'est-à-dire reconnues par les professionnels de la banque. Et ce grâce à la formation, aux fonctions occupées, aux liens professionnels avec les partenaires financiers ou au recrutement de spécialistes ${ }^{14}$. Finalement, ils se «prennent au jeu ». Certains avouent passer du temps avec les chargés de crédit les plus diplômés et se former ainsi aux bases de la gestion du risque. Ils préfèrent dans leur activité les échanges avec les banquiers, ou bien encore se surprennent à lire régulièrement la presse financière. En outre, les banques et les institutions financières partenaires inscrivent quotidiennement le cœur de l'activité de micro-crédit dans des contraintes et des logiques économiques. L'utilisation du jargon financier semble d'ailleurs témoigner de la complexité de ce cadre. Ce contexte contribue à relancer la nécessité de montrer à la fois son adhésion et sa compétence.

Un directeur d'une association de micro-crédits professionnels, responsable de quinze salariés, déclare :

«Non. Je ne voudrais pas bosser dans une banque. Moi j'appartiens au secteur d'activité de la finance et du développement économique, les deux à la fois. Donc, c'est pas si éloigné que ça de la banque mais

\footnotetext{
${ }^{14}$ Le développement des réseaux conduit rapidement au recrutement de spécialistes de la finance ou, plus précisément, de responsables du montage financier des dossiers de prêts. Ils vont assumer la part la plus visiblement économique et financière des activités.
} 
moi, personnellement, je ne me verrais pas courir derrière les clients pour leur demander de placer de l'argent sur mes comptes afin d'en fabriquer, je ne sais pas quoi avec d'ailleurs. (...) L'argent n'est qu'un moyen, c'est un magnifique moyen dans le monde d'aujourd'hui et c'est extrêmement valorisant à titre personnel de pouvoir être le directeur d'une association de micro-crédit comme celle-ci, mais ça n'est qu'un moyen. L'enjeu n'est pas là dedans, l'enjeu il est de permettre à des gens dans un bassin d'emploi où il y a $3 \%$ de taux de chômage, (...) de sortir les $3 \%$ qui reste, et bien si l'argent pouvait être l'outil pour ce projet là au bon sens du terme, ça me plaît de faire ce boulot là. (...) Moi je voulais sortir de la relation interpersonnelle. »

Interrogée, une correspondante de la Nef en région, bénévole, retraitée de l'Éducation nationale (professeur de mathématiques en collège et lycée) déclare :

\section{« D'accord, et après tout ça, vous avez fait des études en économie ?»}

"Oui, parce qu'en fait quand je suis allée dans tous les pays où je vous ai dit [elle a beaucoup voyagé grâce à des mises en disponibilité et au moment de sa retraite anticipée et parle de "séjours d'études marquants ", notamment en Israël et aux Comores], je me demandais toujours si l'éducation est nécessaire et suffisante pour tirer ces pays de la misère. Moi, c'est une vraie interrogation. Moi je me suis dit, c'est peut être pas par l'éducation, c'est peut être par l'économie. Moi je trouve qu'on ne quitte pas les bancs de l'école quand on est prof et ça me manquait cette ouverture. Je suis allée en IUT (Institut universitaire de technologie) à Nantes en formation continue pendant trois ans. En GEA - Gestion des entreprises et des administrations. "

Venir du monde de l'éducation ou du social (par l'emploi précédemment occupé, l'engagement associatif, les expériences de stage et/ou la spécialité du dernier diplôme), et en même temps se former en économie, acquérir des compétences, par le frottement quotidien aux banquiers et autres professionnels de la finance, sont autant d'actions qui développent le goût pour l'économie. Ces actions permettent en outre de se positionner comme un spécialiste à part entière d'un secteur spécifique de l'économie, de faire sa place sur un territoire du financier raisonné donc possible.
Pour eux, il n'est pas question, comme ils le déclarent souvent, de s'imposer comme le « banquier des pauvres ». Il s'agit de devenir un acteur reconnu de " l'économie autrement»; et cela passe par l'acquisition et la démonstration d'un savoir d'économiste, voire de financier. La reconnaissance de la compétence professionnelle s'affirme ici comme un enjeu pour ces professionnels du micro-crédit. Déniant leur appartenance au monde bancaire, trop visiblement marchand, ils développent pourtant des savoir-faire de banquier. Ces compétences financières et gestionnaires, reconnues comme telles par les collègues, les pairs, les partenaires financiers, les travailleurs sociaux des institutions relais, permettent aux individus de construire leurs identités professionnelles en dehors du champ de l'action sociale. Ils s'inscrivent ainsi dans l'espace bancaire en revendiquant d'y devenir les figures emblématiques de "l'accès à la banque pour tous $»$.

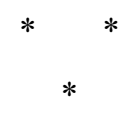

L'objectif était ici de comprendre comment, dans un contexte récent d'organisation des activités de microcrédit, les banquiers solidaires participaient à affirmer, en termes de positionnement et de reconnaissance professionnels et de représentations de leur propre métier, une profession économique bien plus sûrement qu'une profession du social. Nous avons observé ici le processus de constitution de l'espace de la banque solidaire, et ce au plus près des trajectoires sociales, des activités et des discours de ses agents. Cela nous permet de (re)placer les institutions de finances solidaires dans le champ bancaire au sein duquel elles participent à pérenniser un marché, celui du crédit.

Les « gens » du micro-crédit peuvent finalement être apparentés, au niveau de leur technicité notamment, aux " gens de la banque » (Grafmeyer, 1992), aux conseillers commerciaux, aux chargés de clientèle, tant les savoir-faire et les activités des uns et des autres se rejoignent, voire se confondent. La technique financière que suppose le montage d'un dossier de prêt, le calcul du ratio de risque et des garanties nécessaires sont interchangeables. Dans le champ du microcrédit, comme dans celui de la banque (coopérative ou classique), l'activité principale est le financement 
des projets d'une clientèle de particuliers ou d'entreprises. Les taux d'intérêt peuvent varier, les garanties demandées peuvent prendre différentes formes. Pour autant, la pérennisation de l'entreprise réside dans la captation d'emprunteurs potentiels, le financement de projets viables, durables, et la couverture assurée des frais de fonctionnement ; ce dernier aspect conduisant la plupart des structures à rechercher et à obtenir, via la réponse à des appels d'offre, des subventions, pour pérenniser au moins le paiement des salaires.

Et si nous avons parlé ici de « profession économique ", c'est aussi parce que les professionnels concernés, plus parfois même que leurs homologues des agences bancaires traditionnelles, mentionnent leur attrait pour l'activité économique. Ils dessinent ainsi, in fine, les contours du métier de « banquier solidaire » au sein des professions bancaires.

Les structures ont connu des évolutions récentes, tant au niveau des recrutements des salariés que de l'intérêt nouvellement porté par les banques (coopératives dans un premier temps) à cette part jusque-là globalement ignorée du « marché de l'argent ». Ces évolutions traduisent un positionnement de plus en plus affirmé des finances solidaires et de leurs activités professionnelles du côté de l'espace bancaire.

À cet égard, la place prépondérante qu'occupent les chargés de crédit ${ }^{15}$ dans les entreprises de micro-

\footnotetext{
15 Ce terme de « chargé de crédit » n'est pas nécessairement utilisé dans toutes les institutions concernées. Il renvoie à tous les individus dont le travail est centré sur le montage et le suivi financier des dossiers.
}

crédit est révélatrice. En effet, ils forment, au fur et à mesure du développement des établissements, le groupe le plus important en masse salariale, le groupe qui incarne, par nécessité opérationnelle, le cœur du métier. Ils sont également, après l'équipe de direction, les premiers recrutés ; ceux qui, comme le précise un responsable d'un service dédié des Caisses d'Épargne, «font tourner l'activité ».

L'arrivée progressive de certains groupes bancaires français ${ }^{16}$ sur l'espace de la micro-finance (Caisses d'Épargne et leur parcours « confiance » et surtout les partenariats systématiques que les organismes de finances solidaires établissent avec des banques qui, en fait, octroient les prêts) signe le positionnement des uns et des autres sur un marché concurrentiel. La participation, marginale, des groupes bancaires au financement des populations économiquement fragiles ne constitue pas un enjeu directement financier. Certes, même s'ils atteignent des taux de remboursement très élevés, les micro-crédits accordés ne sont pas rentables ${ }^{17}$. Dans un contexte de «moralisation » de l'économie et de l'activité bancaire en particulier, l'enjeu est ici d'ordre symbolique.

\footnotetext{
16 Les banques coopératives s'engagent sur ce terrain du microcrédit avec l'octroi de $80 \%$ de l'ensemble des prêts concédés par les banques. (Source : rapport CDC 2008).

${ }^{17}$ Notons que les clients bénéficiaires des micro-crédits sont très souvent domiciliés dans des banques concurrentes (ainsi seuls $23 \%$ des prêts octroyés au sein du «Parcours confiance » des Pays de la Loire l'ont été à des clients des Caisses d'Épargne). Ces clients sont peu intéressants du point de vue de leur solvabilité et de leur capacité d'emprunt ; cependant, ils augmentent le nombre des emprunteurs dans un contexte fortement concurrentiel où le secteur peine à capter de nouveaux clients.
} 


\section{Bibliographie}

Boltanski L. (1982), Les cadres. La formation d'un groupe social, Paris, Minuit.

Boltanski L., Chiapello E. (1999), Le nouvel esprit du capitalisme, Paris, Gallimard.

Darbus F. (2009) Pratiques et praticiens de l'économie sociale et solidaire (2000-2007). Contribution à la sociologie des croyances économiques, Paris, EHESS. Thèse de sociologie.

Eme B., Laville J.-L, Mothé D., Perret B., Roustang G. (1996), Vers un nouveau contrat social, Paris, Desclée de Brouwer.

Fontaine L. (2008), L'économie morale. Pauvreté, crédit et confiance dans l'Europe préindustrielle, Paris, Gallimard.

Glémain P. (dir.), Bévant D., Bioteau E., Moulévrier P., Taupin M.-T. (2007), Les territoires des finances solidaires : une analyse régionale en Bretagne et Pays de la Loire, DIIESES, juin.

Gloukoviezoff G. (sous la dir. de) (2004), Exclusion et liens financiers. L'exclusion bancaire des particuliers, Économica.

Gloukoviezoff G., Palier J., Lazarus. J (2008), Évaluation d'impact des Crédits Projet Personnel du Secours Catholique, Juin.
Grafmeyer Y. (1992), Les gens de la banque, Paris, PUF, Coll. « Sociologie ».

Hély M. (2008), « Servir l'intérêt général ou produire de l'utilité sociale », Les mondes du travail, ${ }^{\circ} 5$.

Laville J.-L. (1995), « L'économie solidaire : une nouvelle forme d'économie sociale ? ", RECMA, $\mathrm{n}^{\circ} 255$.

Lazuech G. (2006), « Les cadres de l'économie sociale et solidaire : un nouvel entreprenariat ?», Formation Emploi, n $^{\circ}$ 95, pp. 59-74.

Moulévrier P. (2002), Le mutualisme bancaire. Le Crédit Mutuel de l'Église au marché, Rennes, PUR.

Polanyi K. (1983 [1944]), La Grande Transformation. Aux origines politiques et économiques de notre temps, Paris, Gallimard, (traduction française d'après la version originale en anglais) The Great Transformation. The political and economic origins of our time, New York, Farrar \& Rinebart, Paris, Gallimard.

Weber M. (1964), L'éthique protestante et l'esprit du capitalisme, Paris, Plon.

Weber M. (1995), Économie et société, tomes 1 et 2, Paris, Plon. 


\section{Résumé}

\section{Les « banquiers solidaires» ou la légitimation d'une «profession économique»}

\section{Pascale Moulévrier}

Les «banquiers solidaires " constituent un groupe professionnel qui s'autonomise progressivement de la profession de banquier classique. Pour autant, il puise dans le champ économique et financier les ressources pour en devenir une catégorie spécifique. L'ancrage professionnel des individus se caractérise par un chevauchement entre l'espace de la finance, de la banque et celui du travail social. Dès lors, au-delà des parcours, les pratiques professionnelles quotidiennes ne confèrent pas une identité professionnelle et statutaire immédiate. Les porosités entre le contenu solidariste des activités et son aspect financier, entre le salariat et le bénévolat, entre le responsable (rarement appelé directeur), les autres employés et les adhérents (bénéficiaires ou pas) fondent ainsi cet espace professionnel du micro-crédit.

\section{Mots clés}

Métiers des banques-assurances, professionnalisation, économie sociale

Journal of Economic Literature : J 44 